\title{
THE WIELANDT SUBALGEBRA OF A LIE ALGEBRA
}

\author{
DONALD W. BARNES and DANIEL GROVES
}

(Received 9 October 2000; revised 6 February 2002)

\author{
Communicated by R. B. Howlett
}

\begin{abstract}
Following the analogy with group theory, we define the Wielandt subalgebra of a finite-dimensional Lie algebra to be the intersection of the normalisers of the subnormal subalgebras. In a non-zero algebra,this is a non-zero ideal if the ground field has characteristic 0 or if the derived algebra is nilpotent, allowing the definition of the Wielandt series. For a Lie algebra with nilpotent derived algebra, we obtain a bound for the derived length in terms of the Wielandt length and show this bound to be best possible. We also characterise the Lie algebras with nilpotent derived algebra and Wielandt length 2 .
\end{abstract}

2000 Mathematics subject classification: primary 17B30, 17B50.

Keywords and phrases: Lie algebras, subnormal subalgebras.

\section{Introduction}

The Wielandt subgroup of a group $G$, denoted by $\omega(G)$, is the intersection of the normalisers of all the subnormal subgroups of $G$. An automorphism of $G$ permutes the subnormal subgroups, so leaves $\omega(G)$ fixed. Thus $\omega(G)$ is a characteristic subgroup of $G$.

The Wielandt subgroup was first studied by Wielandt [16] in 1958. He proved that, if $G$ is a non-trivial finite group, then $\omega(G)$ is non-trivial. This makes it possible to define the Wielandt series by $\omega_{1}(G)=\omega(G)$ and $\omega_{i+1}(G) / \omega_{i}(G)=\omega\left(G / \omega_{i}(G)\right.$ ) for $i \geq 1$. For a finite group $G$, there is some $n$ such that $\omega_{n}(G)=G$. The least such $n$ is called the Wielandt length of $G$.

There have been a number of investigations of the structure of groups with respect to the Wielandt series (see $[3,4,5,6,7,8])$. In [4], Bryce and Cossey show that a finite soluble group of Wielandt length $n$ has derived length at most $5 n / 3+1$.

The first author made his contribution to this paper while an Honorary Associate of the School of Mathematics and Statistics, University of Sydney.

(C) 2003 Australian Mathematical Society 1446-7887/03 \$A2.00+0.00 
There is a tradition of investigating analogies between group theory and the theory of Lie algebras. There are myriad correspondences between these two fields. In this paper, we develop a theory of the Wielandt series for a finite-dimensional Lie algebra $L$ with nilpotent derived algebra, analogous to the above cited theory for finite groups. As we shall see (Example 5.4 and Example 7.2 below), the restriction $L^{\prime}$ nilpotent is needed. In non-zero characteristic, solubility is not enough.

\section{Preliminaries}

Let $L$ be a finite-dimensional Lie algebra over the field $F$. The Wielandt subalgebra $\omega(L)$ of $L$ is the intersection of the normalisers of the subnormal subalgebras of $L$. The Wielandt series of $L$ is defined by $\omega_{i+1}(L) / \omega_{i}(L)=\omega\left(L / \omega_{i}(L)\right)$ and the Wielandt length of $L$ is the length of this series. This definition of $\omega_{i+1}(L)$, to be meaningful, needs the preceding terms to be ideals. For the definition of the Wielandt length to be meaningful, we need the series to reach $L$, that is, we need $\omega(L) \neq 0$ whenever $L \neq 0$. As we shall see in Section 3, these always hold if the field $F$ has characteristic 0 . In Section 5, we show that they also hold for algebras $L$ whose derived algebra $L^{\prime}$ is nilpotent. We shall make frequent reference to Lie algebras with nilpotent derived algebra, so it is convenient to have a term for them. We adopt the terminology used by Strade and Farnsteiner [14].

DEFINITION 2.1. A Lie algebra $L$ is called completely soluble if its derived algebra $L^{\prime}$ is nilpotent.

All finite-dimensional soluble Lie algebras over a field of characteristic 0 are completely soluble. (For example, see Jacobson [11, page 51].) Thus our results on completely soluble Lie algebras apply to all finite-dimensional soluble Lie algebras over fields of characteristic 0 .

All vector spaces and algebras considered in this paper are assumed to be finitedimensional over a field which we denote by $F$. For any Lie algebra $L$, we denote by $L^{r}$ the terms of the descending central series of $L$, defined by $L^{1}=L$ and $L^{r+1}=\left[L^{r}, L\right]$. We denote by $L^{\infty}$ the intersection of the $L^{r}$ and the centre of $L$ by $\mathscr{Z}(L)$. We denote by $\operatorname{ad}_{u}$ the adjoint derivation of $L$ given by $u \in L$, defined by $\operatorname{ad}_{u}(x)=[u, x]$ for all $x \in L$. We denote the subspace spanned by a set $U$ by $\langle U\rangle$. We write $U<L$ if $U$ is a subalgebra of $L, U \triangleleft L$ if $U$ is an ideal of $L$ and $U \triangleleft L$ if $U$ is a subideal (subnormal subalgebra) of $L$. We denote the normaliser of $U$ in $L$ by $\mathscr{N}_{L}(U)$ and the centraliser by $\mathscr{C}_{L}(U)$. For any subset $U \subseteq L$, we denote by $U^{L}$ the normal closure of $U$ in $L$, that is, the smallest ideal of $L$ which contains $U$. We denote the subnormal closure by $U^{s L}$. 
We define inductively the series of subalgebras $U^{L, i}$ by $U^{L, 0}=L$ and $U^{L, i+1}=U^{U^{L, i}}$. Since $L$ is assumed finite-dimensional, we have $U^{L, i}=U^{s L}$ for all sufficiently large $i$. We will find it convenient to have another description of $U^{s L}$ when $U=\{u\}$ has only one element. Let $X$ be the free Lie algebra over $F$ on the generators $x, x_{1}, x_{2}, \ldots$ We shall regard $X$ as a set of Lie polynomials in the variables $x, x_{1}, x_{2}, \ldots$. Put $X_{i}=X^{\prime} \cap x^{X, i}$. Thus $X_{1}$ is spanned by all products with at least one factor $x$ and $X_{2}$ is spanned by certain of the products with 2 factors $x$. For example, $\left[\left[\left[x_{1}, x\right], x_{2}\right], x\right] \in X_{2}$ but $\left[\left[\left[x_{1}, x\right], x\right], x_{2}\right] \notin X_{2}$. For any Lie algebra $L$ and $u, a_{1}, \ldots \in L$, we have the substitution homomorphism $X \rightarrow L$ in which $x \mapsto u$ and $x_{i} \mapsto a_{i}$ for all $i$. This maps each polynomial in $X_{i}$ into $L^{\prime} \cap u^{L, i}$. We denote by $X_{i}(u, L)$ the space spanned by all the elements so obtained from all possible choices of the $a_{i}$. Clearly, $u^{L, i}=\left\langle u, X_{i}(u, L)\right\rangle$ and, if $u \notin L^{\prime}, X_{i}(u, L)=L^{\prime} \cap u^{L, i}$.

\section{The special case of characteristic 0}

When the field $F$ has characteristic 0 , we have available Tuck's Theorem [15].

THEOREM 3.1. Let $L$ be a finite-dimensional Lie algebra over a field of characteristic 0 , and let $G$ be the group of all automorphisms of $L$. If $H$ is a subalgebra of $L$ which is invariant under $G$, then $H$ is a characteristic ideal of $L$.

Tuck's Theorem is important since it means that any 'natural' subalgebra will be a characteristic ideal, and in particular, $\omega(L)$ is a characteristic ideal. We shall also use the fundamental result of Schenkman [12]:

THEOREM 3.2. Let $L$ be a finite-dimensional Lie algebra over any field and let $U \triangleleft L$. Then $U^{\infty} \triangleleft L$.

We now show that the theory of the Wielandt series in characteristic 0 essentially reduces to that for soluble algebras. We denote the (soluble) radical of $L$ by $R(L)$.

THEOREM 3.3. Let $L$ be a finite-dimensional Lie algebra over a field $F$ of characteristic 0. Then $\omega(L)=\bigcap_{U \triangleleft \triangleleft(L)} \mathscr{N}_{L}(U)$ and $\omega(L) \cap R(L)=\omega(R(L))$.

Proof. If $U \triangleleft L$, then $R(U)=U \cap R(L)$, and it follows that if $U \triangleleft L$, then $R(U)=U \cap R(L)$. Let $U \triangleleft \Delta$ and let $S$ be a Levi factor of $U$. Put $R_{0}=R(U)$. Let $x \in \mathscr{N}_{L}(U)$, and put $R_{1}=[U, R(U)]$. Then $x$ normalises $R_{1}$. Since $\left(S+R_{1}\right) / R_{1}$ is a characteristic ideal of $U / R_{1}=\left(S+R_{1}\right) / R_{1} \oplus R(U) / R_{1}, x$ normalises $S+R_{1}$. Conversely, any $x$ which normalises both $U_{1}:=S+R_{1}$ and $R_{0}$ also normalises $U$. Defining $R_{i+1}=\left[U_{i}, R_{i}\right]$ and $U_{i+1}=S+R_{i+1}$, we see that any $x$ which normalises $U_{i}$ and all the $R_{i}$ also normalises $U$. However, for some $n$, we have $\left[U_{n}, R_{n}\right]=R_{n}$ 
and $\left(U_{n}\right)^{\infty}=U_{n}$. Therefore $U_{n} \triangleleft L$ and any element $x \in L$ which normalises all the $R_{i}$ also normalises $U$. Thus any element $x \in L$ which normalises every subnormal subalgebra of $R$ also normalises every subnormal subalgebra of $L$.

In [10], Hartley proved that, if $L$ is a soluble Lie algebra over a field of characteristic 0 and if $A$ is a minimal ideal of $L$, then $A \leq \omega(L)$. (In Section 5 below, we shall prove this for completely soluble algebras $L$ over any field.) Since for a semi-simple algebra $S, \omega(S)=S$, it follows for algebras over a field of characteristic 0 , that $L \neq 0$ implies $\omega(L) \neq 0$. Thus the Wielandt series reaches $L$ and the Wielandt length is defined. From Theorem 3.3, it follows that $\omega_{i}(L) \cap R(L)=\omega_{i}(R(L))$. It follows that the Wielandt length of $L$ is either equal to or one more than the Wielandt length of $R(L)$.

\section{Subnormal subalgebras in characteristic $p$}

In [12], Schenkman also proved that, if char $F=0$ and if $A, B \triangleleft L$, then their join $A \cup B \triangleleft L$. His proof depends on two special features of Lie algebras in characteristic 0 , namely that if $R=R(L)$, then $L / R$ is a direct sum of simple algebras and that $R^{\prime}$ is nilpotent. In non-zero charactistic, his proof works if we restrict ourselves to completely soluble Lie algebras. We begin with a useful result which is implicit in Schenkman's proof.

LEMMA 4.1. Let $U$ be a nilpotent subnormal subalgebra of the completely soluble Lie algebra $L$, and let $N$ be the nil radical of $L$. Then $U \leq N$.

Proof. Since $U \triangleleft L, U$ acts nilpotently on the $U$-module $L / U$. As $U$ is nilpotent, it acts nilpotently on $U$, and so on $L$. It follows that every chief factor of $U+N$ is central, so $U+N$ is nilpotent. But $N \geq L^{\prime}$, so $U+N \triangleleft L$ and therefore $U+N \leq N$.

LEMMA 4.2. Suppose $L$ is completely soluble and that $A \triangleleft L$ and $B \triangleleft L$. Then $A \cup B \bowtie L$.

Proof. If there exists an ideal $K \triangleleft L, K \leq A$, then we can work in $L / K$ and the result follows by induction over the dimension of $L$. If no such $K$ exists, then by Theorem 3.2, $A$ is nilpotent. Thus we may assume both $A$ and $B$ nilpotent. By Lemma 4.1, both $A$ and $B$ are in the nil radical $N$. It follows that $A \cup B \triangleleft N \triangleleft L$.

The condition that $L$ is completely soluble is needed in Lemma 4.2. Assuming $L$ soluble is not enough as is shown by the following example. 
EXAMPLE 4.3. Let $V=\left\langle v_{0}, \ldots, v_{p-1}\right\rangle$ where $p=\operatorname{char} F$ and the indices are integers $\bmod p$. Let $L=\langle x, y, z, V\rangle$ with multiplication defined by $[x, y]=z$, $[x, z]=[y, z]=\left[v_{i}, v_{j}\right]=0$, and $\left[x, v_{n}\right]=n v_{n-1},\left[y, v_{n}\right]=v_{n+1},\left[z, v_{n}\right]=v_{n}$. Let $A=\langle x\rangle$ and $B=\langle y-z\rangle$. Then $A \triangleleft L, B \triangleleft L$ but $A \cup B=\langle x, y, z\rangle$ is not subnormal in $L$.

Proof. Since $L / V$ is nilpotent, both $A+V$ and $B+V$ are subnormal in $L$. As $x$ acts nilpotently on $V, A+V$ is nilpotent, so $A \triangleleft \triangleleft(A+V) \triangleleft \Delta . V$ is an irreducible $L / V$-module. By Zassenhaus [17, Lemma 1], all the composition factors of $V$ as $B$-module are isomorphic. Now $\left[(y-z),\left(v_{0}+\cdots+v_{p-1}\right)\right]=0$. It follows that $(y-z)$ acts nilpotently on $V$. Hence $B+V$ is nilpotent and it follows that $B \triangleleft L$. Since $A \cup B$ is nilpotent, if $A \cup B \bowtie L$, then every subalgebra of $A \cup B$ is subnormal in $L$, in particular $\langle z\rangle$, is subnormal. But $\langle z\rangle$ is not subnormal in $\langle z\rangle+V$. Therefore $A \cup B$ is not subnormal.

\section{Some basic properties of the Wielandt subalgebra}

In this section, we establish some basic properties of the Wielandt subalgebra $\omega(L)$ of a completely soluble Lie algebra $L$.

THEOREM 5.1. Let $L$ be a soluble Lie algebra and let $w \in \omega(L)$. Then there exists $\lambda \in F$ such that for all nilpotent subalgebras $U \bowtie L$ and all $u \in U,[w, u]=\lambda u$. If for any such $U, U^{\prime} \neq 0$ or $U \notin L^{\prime}$, then $\lambda=0$.

Proof. Let $u \in U \triangleleft \Delta L$ and suppose $U$ is nilpotent. Then $\langle u\rangle \triangleleft \Delta$. Since $w \in$ $\mathscr{N}_{L}\langle u\rangle,[w, u]=\lambda_{u} u$ for some $\lambda_{u} \in F$. We have to show that $\lambda_{u}$ is independent of the choice of $u$. Let $A$ be a minimal ideal of $L$ and let $a \in A, a \neq 0$. Since $\langle u\rangle \triangleleft \Delta$, $\operatorname{ad}_{u}^{r}(A) \leq\langle u\rangle$ for some $r$ and $\operatorname{ad}_{u}^{r+1}(A)=0$. It follows that $\langle u\rangle+A$ is nilpotent and subnormal. Therefore $\lambda_{u+a}(u+a)=[w, u+a]=[w, u]+[w, a]=\lambda_{u} u+\lambda_{a} a$. It follows that $\lambda_{u}=\lambda_{a}$.

Now suppose we have $U \triangleleft \Delta, U$ nilpotent and $U^{\prime} \neq 0$. Take $u, v \in U$ with $x=[u, v] \neq 0$. Then

$$
\lambda x=[w,[u, v]]=[[w, u], v]+[u,[w, v]]=[\lambda u, v]+[u, \lambda v]=2 \lambda x,
$$

so $\lambda=0$.

Suppose $U \triangleleft L, U$ nilpotent and $U \notin L^{\prime}$. Take $u \in U, u \notin L^{\prime}$. We then have $\lambda u=[w, u] \in L^{\prime}$, so $\lambda=0$.

COROLlaRY 5.2. Let $N$ be a nilpotent Lie algebra. Then $\omega(N)=\mathscr{Z}(N)$. 
PROof. If $w \in \omega(N)$ and $N$ is non-abelian, then $w \in \mathscr{Z}(N)$ by Theorem 5.1.

LEMMA 5.3. Suppose $L$ is completely soluble and let $A$ be a minimal ideal of $L$. Then $A \leq \omega(L)$.

PROOF. Since $A$ is a minimal ideal, either $A \cap L^{\prime}=A$ or $A \cap L^{\prime}=0$. If $A \cap L^{\prime}=0$, then for all $x \in L$ and $a \in A$, we have $[x, a] \in A \cap L^{\prime}=0$ so $a \in \mathscr{Z}(L) \leq \omega(L)$. Suppose $A \leq L^{\prime}$. Let $U \triangleleft L$. We show that $A \leq \mathscr{N}_{L}(U)$. This holds if $U \triangleleft L$, so suppose $U$ is not an ideal. There exists a maximal ideal $M>U$. But $M \geq L^{\prime}$. Hence $U+L^{\prime}$ is a proper ideal of $L$. Since $L^{\prime}$ is nilpotent, $A \leq \mathscr{Z}\left(L^{\prime}\right)$ and we can consider $A$ as an irreducible module for the abelian algebra $L / L^{\prime}$. As $\left(U+L^{\prime}\right) / L^{\prime}$-module, $A$ is a direct sum of copies $A_{i}$ of an irreducible submodule $A_{1}$. Now $A_{i}$ is a minimal ideal of $U+L^{\prime}$, so by induction over $\operatorname{dim} L$, we have $A_{i} \leq \mathscr{N}_{U+L^{\prime}}(U) \leq \mathscr{N}_{L}(U)$. Thus $A=\oplus_{i} A_{i} \leq \mathscr{N}_{L}(U)$.

The condition that $L$ is completely soluble is needed in Lemma 5.3.

EXAMPLE 5.4. Let $L$ be the algebra of Example 4.3 above. We have $A+V$ and $B+V$ both nilpotent, subnormal and not in $L^{\prime}$. By Theorem 5.1, $\omega(L) \leq \mathscr{C}_{L}(A+V)$ and $\omega(L) \leq \mathscr{C}_{L}(B+V)$. Since $A+V$ and $B+V$ generate $L, \omega(L) \leq \mathscr{Z}(L)=0$.

By Theorem 5.1, for each $w \in \omega(L)$, we have $\lambda(w) \in F$ such that $[w, u]=\lambda(w) u$ for every element $u$ which lies in some nilpotent subnormal subalgebra. This is clearly a linear map $\lambda: \omega(L) \rightarrow F$.

LEMMA 5.5. Suppose $L$ is completely soluble. Let $N=N(L)$ be the nil radical of L. Let $w \in \omega(L)$. If $w \in N$ then $\lambda(w)=0$ and $w \in \mathscr{Z}(N)$. Conversely, if $\lambda(w)=0$, then $w \in N$.

Proof. Suppose $w \in N$. Let $z \in \mathscr{Z}(N), z \neq 0$. Then $\lambda(w) z=[w, z]=0$, so $\lambda(w)=0$ and for all $x \in N$, we have $[w, x]=\lambda(w) x=0$ and $w \in \mathscr{Z}(N)$.

Suppose conversely that $\lambda(w)=0$. Then $\langle w\rangle+N$ is nilpotent. As $\langle w\rangle+N \geq L^{\prime}$, $\langle w\rangle+N \triangleleft L$, so $\langle w\rangle+N \leq N$, that is, $w \in N$.

We shall have occasion later to consider the algebra direct sum $L \oplus A$ of $L$, assumed completely soluble, with an abelian algebra $A$. Since clearly $A$ normalises every subnormal subalgebra of $L$, it is tempting to expect $\omega(L \oplus A)=\omega(L) \oplus A$, but this is not always true as there are subnormal subalgebras of $L \oplus A$ which do not lie in either direct summand. Indeed, if $\omega(L)$ is non-abelian, then so is $\omega(L) \oplus A$, but $\omega(L \oplus A)$ is abelian by Theorem 5.1 and Lemma 5.5. If however, $\omega(L)$ is abelian, then the expected result holds. More generally, we have the following result. 
THEOREM 5.6. Suppose $A$ and $B$ are non-trivial completely soluble Lie algebras. Let $L$ be the algebra direct sum $L=A \oplus B$. Then $\omega(L)=N(\omega(A)) \oplus N(\omega(B))$ and $\omega(L)$ is abelian.

Proof. Let $a \in A$ and $b \in B$. Suppose $U \triangleleft A$. Then $(a+b) \in \mathscr{N}_{L}(U)$ if and only if $a \in \mathscr{N}_{A}(U)$. Thus $\omega(L) \leq \omega(A) \oplus \omega(B)$. It follows that $N(\omega(L)) \leq$ $N(\omega(A)) \oplus N(\omega(B))$. Now suppose $a \in N(\omega(A))$ and $b \in N(\omega(B))$. We have to show that $a+b \in \omega(L)$. Let $u=c+d$ where $c \in A$ and $d \in B$. Put $U=u^{s L}$, $V=c^{s A}$ and $W=d^{s B}$. We have $U=\left\langle u, X_{i}(u, L)\right\rangle$ for some $i$. We have to show that $[a+b, u] \in U$. But $X_{i}(u, L)=X_{i}(c, A)+X_{i}(d, B)$ and $V=\left\langle c, X_{i}(c, A)\right\rangle$. Thus $[a, c] \in X_{i}(c, A),\left(0\right.$ if $\left.c \in A^{\prime}\right)$ and similarly $[b, d] \in X_{i}(d, B)$. Hence $a+b \in \omega(L)$.

If $a+b \in \omega(L)$, then $[a+b, c+d]=\lambda c+\lambda d$ for all $c \in N(A)$ and all $d \in N(B)$ and some $\lambda \in F$. If $\omega(A)$ is abelian, then $\lambda=0$ and so $a+b \in N(L)$ by Lemma 5.5. Thus $\omega(L)$ is abelian if $\omega(A)$ is abelian.

Now suppose $\omega(L)$ is non-abelian. Then $\omega(A)$ and, by symmetry, $\omega(B)$ are nonabelian. There exists $a+b \in \omega(L)$ such that $[a+b, v]=v$ for all $v \in N(L)$. Since $A \neq A^{\prime}$, there exists $c \in A, c \notin A^{\prime}$. Since $B$ is non-abelian, we can take $d \in B^{\prime}$, $d \neq 0$, and put $u=c+d$. As $\langle d\rangle \triangleleft L,[a+b, d]=d$. Since $a+b \in \omega(L)$, $[a+b, u]=[a, c]+d \in u^{s L}$. But $u^{s L}=\left\langle c+d, X_{i}(c, A)\right\rangle$ for some $i$. Thus $X_{i}(c, A)$ has codimension 1 in $u^{s L}$ whence it follows that $d \notin u^{s L}$. Since $a \in \omega(A)$, $[a, c] \in X_{i}(c, A)$ contrary to $[a, c]+d \in u^{s L}$. Thus $\omega(L)$ is abelian and it follows that $\omega(L)=N(\omega(A)) \oplus N(\omega(B))$.

Since $(A \oplus B) / \omega(A \oplus B) \simeq A / N(\omega(A)) \oplus B /(N(\omega(B))$, we can readily determine $\omega_{i}(A \oplus B)$ by repeated application of Theorem 5.6 with the help of Theorem 6.2 below to deal with any non-abelian cases which arise.

\section{Non-abelian Wielandt subalgebras}

In this section, we determine the structure of completely soluble Lie algebras $L$ with $\omega(L)$ non-abelian.

LEMMA 6.1. Suppose $L$ is completely soluble. Then $\omega(L)$ is abelian if and only if the map $\lambda$ is the zero map.

Proof. If $\lambda=0$, then $\omega(L) \leq \mathscr{Z}(N)$, so $\omega(L)$ is abelian. If $\lambda(w) \neq 0$ and $A$ is a minimal ideal of $L$, then $\langle w, A\rangle$ is a subalgebra by Theorem 5.1, clearly non-abelian and $\langle w, A\rangle \leq \omega(L)$ by Lemma 5.3.

THEOREM 6.2. Suppose $L$ is completely soluble and $\omega(L)$ is non-abelian. Then $N(L)=L^{\prime}$ is abelian and $L=A \oplus L^{\prime}$ (vector space direct sum) where $A<L$. There 
exists $w \in A \cap \omega(L)$ with $\lambda(w)=1$ and $\omega(L)=\left\langle w, L^{\prime}\right\rangle$ which is a characteristic ideal of $L$. The Wielandt series of $L$ exists and $\omega_{2}(L)=L$. If $c \in A, c \notin\langle w\rangle$ then the linear transformation $\bar{c}: L^{\prime} \rightarrow L^{\prime}$ given by the action of $c$ is invertible and has no eigenvalues in $F$.

ProOF. By Theorem 5.1 and Lemma 6.1, $N(L)=L^{\prime}$ is abelian. There exists $w \in \omega(L)$ with $\lambda(w) \neq 0$, so we may choose $w$ with $\lambda(w)=1$. Put $V=L^{\prime}$. We consider $V$ as an $L / L^{\prime}$-module. We have $V^{L / L^{\prime}}=0$. By Barnes [1, Theorem 1], $H^{2}\left(L / L^{\prime}, V\right)=0$. Thus there exists a subalgebra $A$ which complements $L^{\prime}$ in $L$. Since every minimal ideal of $L$ is contained in $N(L)$ and $A \cap N(L)=0, A$ contains no non-trivial ideal of $L$. Thus $\mathscr{C}_{A}\left(L^{\prime}\right)=0$ and $V$ is a faithful $A$-module.

For any $v \in V$, the map $\alpha_{v}=1-\operatorname{ad}_{v}: L \rightarrow L$ is an automorphism of $L$ since

$$
\begin{aligned}
{\left[\alpha_{v}(x), \alpha_{v}(y)\right] } & =[x-[v, x], y-[v, y]] \\
& =[x, y]-[[v, x], y]-[x,[v, y]]+[[v, x],[v, y]] \\
& =[x, y]-[v,[x, y]]=\alpha_{v}[x, y]
\end{aligned}
$$

as $[v, x],[v, y] \in V$ which is abelian. Let $w^{\prime}$ be the element of the coset $w+L^{\prime}$ which lies in $A$. Then $w^{\prime}=w+v_{0}$ for some $v_{0} \in V$. Since $w^{\prime}=\alpha_{v_{0}}(w), w^{\prime} \in \omega(L)$ and we may replace $w$ with $w^{\prime}$. Thus we obtain $w \in A$ with the required properties. Further, for every $v \in V, w+v=w-[v, w]=\alpha_{v}(w) \in \omega(L)$, so $v \in \omega(L)$ and $\omega(L)=\left\langle w, L^{\prime}\right\rangle$. Let $d$ be any derivation of $L$. Then $d L^{\prime} \subseteq L^{\prime}$. Also, for any $v \in V$, we have $d v=d[w, v]=[d w, v]+[w, d v]=[d w, v]+d v$, so $d w \in \mathscr{C}_{L}\left(L^{\prime}\right)=L^{\prime}$. Thus $d \omega(L) \subseteq \omega(L)$. Since $L / \omega(L)$ is abelian, $\omega_{2}(L)=L$ and $L$ has Wielandt length at most 2 .

Now take $c \in A, c \notin\langle w\rangle$. Then we can decompose $V$ with respect to $\bar{c}$ into the direct sum $V=V_{0} \oplus V_{1}$ where $\bar{c}$ acts nilpotently on $V_{0}$ and invertibly on $V_{1}$. As $A$ is abelian, this decomposition is $A$-invariant, that is, $V_{0}$ and $V_{1}$ are ideals of $L$. Suppose $V_{0} \neq 0$. Put $V_{0}^{i}=\bar{c}^{i} V_{0}$. For some $r$, we have $V_{0}^{r} \neq 0$ but $V_{0}^{r+1}=0$. Let $v \in V_{0}$ and put $u=c+v$. Then

$$
\left\langle u, V_{1}\right\rangle \triangleleft\left\langle u, V_{0}^{r}, V_{1}\right\rangle \triangleleft\left\langle u, V_{0}^{r-1}, V_{1}\right\rangle \triangleleft \cdots \triangleleft\left\langle u, V_{0}, V_{1}\right\rangle \triangleleft L
$$

and $\left\langle u, V_{1}\right\rangle \bowtie L$. But $[w, u]=[w, c]+[w, v]=v$, so $v \in\left\langle u, V_{1}\right\rangle \cap V_{0}=0$. Therefore $V_{0}=0$ and $\bar{c}$ is invertible. Since for all $\mu \in F, \mu w-c$ acts invertibly on $V, \bar{c}$ has no eigenvalues in $F$.

\section{Normality of the Wielandt subalgebra}

We are now in a position to prove the main result about the Wielandt subalgebra. 
THEOREM 7.1. Suppose $L$ is completely soluble. Then $\omega(L) \triangleleft L$.

PROOF. By Theorem 6.2, we may suppose $\omega(L) \leq \mathscr{Z}(N)$. We then have

$$
\omega(L)=\bigcap_{U \triangleleft L} \mathscr{N}_{L}(U)=\bigcap_{U \triangleleft L}\left(N \cap \mathscr{N}_{L}(U)\right) .
$$

We consider subnormal subalgebras $U$. If $U$ is not nilpotent, then there exists an ideal $A \triangleleft L, 0 \neq A \leq U$. Now $\mathscr{N}_{L / A}(U / A)=\mathscr{N}_{L}(U) / A$. By induction over $\operatorname{dim} L$,

$$
\bigcap_{U \geq A} \mathscr{N}_{L}(U) \triangleleft L
$$

If $U$ is nilpotent, then $U \leq N$ by Lemma 4.1 , and we have

$$
\bigcap_{U \text { nilp }}\left(N \cap \mathscr{N}_{L}(U)\right)=\mathscr{Z}(N) \triangleleft L .
$$

Hence

$$
\omega(L)=\bigcap_{U \triangleleft L} \mathscr{N}_{L}(U)=\bigcap_{A \triangleleft L}\left(\bigcap_{U \geq A} \mathscr{N}_{L}(U)\right) \cap \bigcap_{U \text { nilp }} \mathscr{N}_{L}(U) \triangleleft L .
$$

The following example shows that the condition that $L$ is completely soluble cannot be omitted from Theorem 7.1.

EXAMPLE 7.2. Let $V=\left\langle v_{0}, \ldots, v_{p-1}\right\rangle$ where $p=$ char $F$ and the subscripts are integers $\bmod p$. Put $L=\langle a, b, c, V\rangle$ with multiplication given by $[a, b]=b$, $[a, c]=-c,[b, c]=0,\left[v_{i}, v_{j}\right]=0$ and $\left[a, v_{i}\right]=i v_{i},\left[b, v_{i}\right]=v_{i+1}$ and $\left[c, v_{i}\right]=$ $v_{i-1}$. Then $\omega(L)=\left\langle v_{0}+\cdots v_{p-1}\right\rangle$ which is not an ideal of $L$.

PROOF. We determine the subnormal subalgebras $U$ which are not ideals. Any such $U$ is contained in some maximal ideal which must contain $L^{\prime}$. But $L^{\prime}=\langle b, c, V\rangle$ is a maximal ideal, so $U \leq L^{\prime}$ and so $U \leq M$ for some maximal ideal $M$ of $L^{\prime}$. As $L^{\prime \prime}=V$, $M=\langle\lambda b-\mu c, V\rangle$ for some $\lambda, \mu \in F$. If $\lambda=0$ or $\mu=0$, then $M^{\prime}=V$ and it follows that $U \leq V$. If $\lambda \neq 0$, we may take it to be 1 . Then we have $\left[b-\mu c, v_{i}\right]=v_{i+1}-\mu v_{i-1}$. Thus for all $i$, we have $v_{i}-\mu v_{i-2} \in M^{\prime}$. It follows that $v_{i}-\mu^{r} v_{i-2 r} \in M^{\prime}$ for all $r$. Taking $r=p$, we get $\left(1-\mu^{p}\right) v_{i} \in M^{\prime}$. Thus $M^{\prime}=V$ and $U \leq V$ unless $\mu^{p}=1$, that is, unless $\mu=1$ and $M=\langle b-c, V\rangle$. Since $\langle b-c\rangle \triangleleft\langle a, b, c\rangle$ and $V$ is an irreducible $\langle a, b, c\rangle$-module, it contains only one type of composition factor as $\langle b-c\rangle$-module. As $\left[b-c, v_{0}+\cdots+v_{p-1}\right]=0, b-c$ acts nilpotently on $V$ and $M=\langle b-c, V\rangle$ is nilpotent. Thus $\omega(L)=\mathscr{Z}(M)=\left\langle v_{0}+\cdots+v_{p-1}\right\rangle$. 
If char $F=0$, then $\omega(L)$ is a characteristic ideal. It is natural to ask if this holds for completely soluble Lie algebras $L$ over a field of non-zero characteristic. Suppose $L$ is a minimal counterexample. If $U \triangleleft L$ is not nilpotent, then $U^{\infty}$ is a non-trivial characteristic ideal. It follows that the intersection of the $\mathscr{N}_{L}(U)$ of the non-nilpotent $U \triangleleft \Delta$ is a characteristic ideal of $L$. Let $d$ be any derivation of $L$ and let $M$ be the split extension of $L$ by $d$. Then $d(N)+L^{\prime} \leq M^{\prime}$ and is an ideal of $L$. Now $M$ is soluble. If $M^{\prime}$ is nilpotent, then $M^{\prime} \leq N$, so $d(N) \subseteq N$. It then follows that $d(\mathscr{Z}(N)) \subseteq \mathscr{Z}(N)$ and so $d(\omega(L)) \subseteq \omega(L)$. Hence our minimal counterexample must have a derivation $d$ such that $M^{\prime}$ is not nilpotent. We are now in a position to construct such an example. We have incidently given another proof that $\omega(L)$ is a characteristic ideal if $L$ is a soluble algebra over a field of characteristic 0 as then, $M^{\prime}$ is always nilpotent.

EXAMPLE 7.3. Suppose char $F=p \neq 0$. Let $L=\left\langle y, z, v_{0}, \ldots, v_{p-1}\right\rangle$, where the subscripts are integers $\bmod p$, be the Lie algebra with multiplication given by $[y, z]=0,\left[y, v_{i}\right]=i v_{i+1},\left[z, v_{i}\right]=v_{i}$ and $\left[v_{i}, v_{j}\right]=0$. Then $\omega(L)$ is not a characteristic ideal of $L$.

PROof. We have $N=\left\langle y, v_{0}, \ldots v_{p-1}\right\rangle>L^{\prime}$, so $\omega(L) \leq \mathscr{Z}(N)=\left\langle v_{0}\right\rangle$. As $\left\langle v_{0}\right\rangle$ is a minimal ideal, it follows that $\omega(L)=\left\langle v_{0}\right\rangle$. Let $d: L \rightarrow L$ be given by $d(y)=z$, $d(z)=0$ and $d\left(v_{i}\right)=v_{i-1}$. Then $d$ is a derivation of $L$, (the split extension $M$ of $L$ by $d$ is a familiar example of a soluble algebra with non-nilpotent derived algebra) and $d(\omega(L))=\left\langle v_{p-1}\right\rangle \nsubseteq \omega(L)$.

\section{Wielandt and derived lengths}

A $T$-algebra is a Lie algebra in which the relation of normality is transitive, thus an algebra in which every subideal is an ideal, or in the terminology we have introduced, an algebra of Wielandt length 1 . We require the following theorem of Stewart [13] giving the structure of such algebras:

THEOREM 8.1. $L$ is a soluble $T$-algebra if and only if $L$ is either abelian or the split extension of an abelian algebra by its algebra of scalar linear transformations.

Proof. Let $L$ be a soluble $T$-algebra. We use induction over $\operatorname{dim}(L)$. Since $L$ is soluble, there exists a 1-dimensional subnormal subalgebra $A$ of $L$. Since $L$ is a $T$-algebra, $A \triangleleft L$. As $L / A$ is also a $T$-algebra, by induction, we have that any ideal of $L / A$ of codimension 1 is abelian. Now consider $A$ as an $L$-module. Then $L / \mathscr{C}_{L}(A)$ has dimension at most 1 and it follows that there exists $N \triangleleft L$ of codimension 1 in $L$, such that $A \leq N \leq \mathscr{C}_{L}(A)$. We have $N / A$ abelian and $A \leq \mathscr{Z}(N)$. Thus $N$ is 
nilpotent. Take $x \in L, x \notin N$. Since $L=\omega(L)$, by Theorem 5.1, for some $\lambda \in F$, we have $[x, n]=\lambda n$ for all $n \in N$.

If $\lambda=0$, then $x \in \mathscr{Z}(L)$ and $L$ is nilpotent. We then have $L=\omega(L)=\mathscr{Z}(L)$ by Corollary 5.2 and $L$ is abelian.

If $\lambda \neq 0$, then $N$ is abelian by Theorem 5.1 and $L$ has the asserted structure.

Conversely, suppose $L$ is the split extension of an abelian algebra $A$ by its algebra of scalar linear transformations. Then $A=L^{\prime}$ and has codimension 1 . Thus $A$ is the only maximal ideal of $L$, so every proper subideal is a subalgebra of $A$. Clearly, every subspace of $A$ is an ideal of $L$. Thus $L$ is a $T$-algebra.

If $L$ is completely soluble, $\omega(L) \triangleleft L$ and so every subideal of $\omega(L)$ is also a subideal of $L$, so normalised by $\omega(L)$. Thus $\omega(L)$ is a $T$-algebra. By Theorem $8.1, N(\omega(L))$ is either equal to $\omega(L)$ or is an abelian ideal of it of codimension 1 . In either case, it is a characteristic ideal of $\omega(L)$ and so is an ideal of $L$.

LEMMA 8.2. Suppose L is completely soluble. Then

$$
\left[L^{\prime}, N(\omega(L))\right]=0 \quad \text { and } \quad L^{\prime} \cap \omega(L) \leq N(\omega(L))
$$

Proof. Let $x \in L^{\prime}$. If $x \in N(\omega(L))$, then $[x, N(\omega(L))]=0$ since $N(\omega(L))$ is abelian. Suppose $x \notin N(\omega(L))$. Since $L^{\prime}$ is nilpotent, every subalgebra of $L^{\prime}$ is subnormal in $L$, in particular, $\langle x\rangle \triangleleft L$ and is normalised by $\omega(L)$. Thus $[x, N(\omega(L))] \subseteq\langle x\rangle$. But $N(\omega(L)) \triangleleft L$, so $[x, N(\omega(L))] \subseteq N(\omega(L))$. Thus $[x, N(\omega(L))] \subseteq\langle x\rangle \cap N(\omega(L))=0$ since $x \notin N(\omega(L))$. Thus $\left[L^{\prime}, N(\omega(L))\right]=0$. As $L^{\prime} \cap \omega(L)$ is a nilpotent ideal of $\omega(L)$, it is contained in $N(\omega(L))$.

LEMMA 8.3. If $L$ is completely soluble and has Wielandt length $k$, then $L$ ' has class at most $k$.

PROOF. If $k=1$, then $L$ is a $T$-algebra and $L^{\prime}$ is abelian. Suppose $k>1 . L / \omega(L)$ has Wielandt length $k-1$, so using induction over $k$, we may suppose $(L / \omega(L))^{\prime}$ has class at most $k-1$. Thus $\left(L^{\prime}\right)^{k} \leq \omega(L)$. Now using Lemma 8.2, we have

$$
\left(L^{\prime}\right)^{k+1}=\left[L^{\prime},\left(L^{\prime}\right)^{k}\right] \subseteq\left[L^{\prime}, L^{\prime} \cap \omega(L)\right] \subseteq\left[L^{\prime}, N(\omega(L)]=0 .\right.
$$

THEOREM 8.4. Suppose $L$ is completely soluble. Let $k$ be the Wielandt length of $L$ and let $l$ be the least integer greater than or equal to $\log _{2}(k+1)$. Then $L$ has derived length at most $l+1$.

ProOF. For any $n$, we have $L^{(n+1)}=\left(L^{\prime}\right)^{(n)} \leq\left(L^{\prime}\right)^{2^{n}}$. But $\left(L^{\prime}\right)^{k+1}=0$. Thus $L^{(n+1)}=0$ if $2^{n} \geq k+1$, that is, if $n \geq \log _{2}(k+1)$. 
We now show that this bound is best possible. To do this, we first consider some nilpotent algebras with some special structure.

Let $N$ be a positively graded finite-dimensional Lie algebra over the field $F$. Thus $N=N_{1} \oplus N_{2} \oplus \cdots \oplus N_{k}$ as vector space and $\left[N_{i}, N_{j}\right] \subseteq N_{i+j}$. Then $N$ is nilpotent of class at most $k$. To simplify notation, we put $N_{r}^{*}=\sum_{i \geq r} N_{i}$. We shall suppose that $N$ is generated by $N_{1}$. In this case, if $N_{k} \neq 0, N$ has class precisely $k$, the descending central series of $N$ being $N=N_{1}^{*} \triangleright N_{2}^{*} \triangleright \ldots \triangleright N_{k}^{*} \triangleright N_{k+1}^{*}=0$. The terms $\mathscr{Z}_{i}(N)$ of the ascending central series of $N$ satisfy $\mathscr{Z}_{i}(N) \supseteq N_{k-i+1}^{*}$. To make an inductive argument using the quotient by the centre work smoothly, we suppose that $\mathscr{Z}_{i}(N)=N_{k-i+1}^{*}$ for all $i$. Such an algebra, one in which the terms of the ascending central series are as small as possible, we shall call mini-central. Such algebras do exist as is shown by the following example.

EXAMPLE 8.5. Let $N$ be the Lie algebra of strictly upper triangular $(k+1) \times(k+1)$ matrices. Put $N_{r}=\left\langle e_{i, i+r} \mid i=1, \ldots, k-r+1\right\rangle$ where $e_{i j}$ denotes the matrix with 1 in the $i j$ position and all other entries 0 . This algebra is clearly mini-central.

A mini-central graded nilpotent algebra $N$ has a derivation $d: N \rightarrow N$ of degree 0 given by $d a=i a$ for $a \in N_{i}$. Let $L=\langle d, N\rangle$ be the split extension of $N$ by $\langle d\rangle$. Thus $[d, a]=i a$ for $a \in N_{i}$.

LEMMA 8.6. Let $L$ be as above with $k>1$. Then $\omega(L)=N_{k}$.

PROOF. Since $L$ is soluble, any maximal ideal of $L$ must contain the derived subalgebra $L^{\prime}$. But $L^{\prime}=N$. Thus $N$ is the only maximal ideal and the subnormal subalgebras of $L$ are precisely the subalgebras of $N$. From Theorem 5.1, it follows that $\omega(L)=\mathscr{C}_{L}(N)$. We have only to show that $\omega(L) \leq N$ and the result follows.

Suppose $w \in \omega(L)$ but $w \notin N$. Then without loss of generality, we may suppose $w=d+a$ for some $a \in N$. If $a \notin \mathscr{Z}(N)$, then there exists $b \in N_{r}$ for some $r$ with $[a, b] \neq 0$. We then have $[w, b]=r b+[a, b] \notin\langle b\rangle$ contrary to $w \in \omega(L)$. If $a \in \mathscr{Z}(N)$, we take non-zero elements $b \in N_{1}$ and $c \in N_{2}$. Then $[w, b+c]=$ $b+2 c \notin\langle b+c\rangle$ contrary to assumption.

THEOREM 8.7. The algebra $L$ constructed above has Wielandt length $k$ and derived length $l+1$ where $l$ is the least integer greater than or equal to $\log _{2}(k+1)$ so attaining the bound given in Theorem 8.4. Hence that bound is best possible.

Proof. We take $L$ the algebra constructed as above from the minicentral graded nilpotent algebra $N$ of class $k$. From Lemma 8.6, it follows that $\omega_{k-1}(L)=N_{2}^{*}$. As clearly $\omega\left(L / N_{2}^{*}\right)=L / N_{2}^{*}$, the Wielandt length of $L$ is $k$. The derived length of $N$ is $l$, so the derived length of $L$ is $l+1$. 


\section{Lie algebras with Wielandt length 2}

In this section, we describe the structure of the completely soluble Lie algebras which have Wielandt length precisely 2 . We divide them into three cases according to which, if any, of $\omega(L)$ and $L / \omega(L)$ are non-abelian, both non-abelian being excluded by Theorem 6.2. We have already given the structure for the case $\omega(L)$ non-abelian in Theorem 6.2. We now consider the cases in which $\omega(L)$ is abelian. Some are obtained as direct sums $L=M \oplus A$ where $\omega(M)$ is non-abelian and $A$ is an abelian algebra. The structure of these follows from Theorem 6.2. If $L$ is an algebra with $\omega(L)$ abelian and $A$ is abelian, then $\omega(L \oplus A)=\omega(L) \oplus A$ and $(L \oplus A) / \omega(L \oplus A) \simeq L / \omega(L)$. Thus we need only consider algebras $L$ which have no non-trivial abelian direct summand.

THEOREM 9.1. Suppose char $F \neq 2$. Let L be a completely soluble Lie algebra over $F$ with Wielandt length 2 . Suppose $L / \omega(L)$ is non-abelian. Suppose $L$ has no non-trivial abelian direct summand. Then there exist subspaces $W, A$ and an element $b$ such that the following conditions are satisfied.

(1) As vector space, $L=W \oplus A \oplus\langle b\rangle$.

(2) $[W, W+A]=0$.

(3) Setting $\beta(w)=[w, b]$ defines an invertible linear map $\beta: W \rightarrow W$.

(4) Put $W_{1}=\left\{w \in W \mid(\beta-1)^{n} w=0\right.$ for some $\left.n\right\}$. Then for all $a \in A$, $[a, b]=a+\mu(a)$ where $\mu(a) \in W_{1}$.

(5) $[A, A]=L^{\prime \prime} \leq W$ and $[w, b]=2 w$ for all $w \in L^{\prime \prime}$.

(6) For all $a \in A, a \neq 0$, there exists $a^{\prime} \in A$ with $\left[a, a^{\prime}\right] \neq 0$.

Proof. Put $W=\omega(L)$. Then by assumption, $[W, W]=0$. Also $L^{\prime} \nless W$. We choose some subspace $A$ complementary to $W$ in $L^{\prime}+W$. Then $(L / W)^{\prime}=$ $(A+W) / W$. Since $L / W$ is non-abelian of Wielandt length $1, A+W$ has codimension 1 in $L$, and there exists $b \in L$ such that $[a, b]=a+\mu(a)$ with $\mu(a) \in W$ for all $a \in A$. Also $[A, A] \subseteq W$. Let $f(x)=x^{r}(x-1)^{s} g(x)$, where $x$ and $(x-1)$ do not divide $g(x)$, be the minimum polynomial of the linear map $\beta: W \rightarrow W$ given by $\beta(w)=[w, b]$ for all $w \in W$. Put $W_{0}=(\beta-1)^{s} g(\beta) W, W_{1}=\beta^{r} g(\beta) W$ and $W_{*}=\beta^{r}(\beta-1)^{s} W$. Then $\beta$ is nilpotent on $W_{0}$ and invertible on $W_{1}+W_{*}$. We now adjust our choice of $A$. For any linear map $\alpha: A \rightarrow W$, we can replace $A$ by $\{a+\alpha(a) \mid a \in A\}$. Observe that if $a_{1}=a+\alpha(a)$, then

$$
\left[a_{1}, b\right]=[a, b]+[\alpha(a), b]=a+\mu(a)+\beta \alpha(a)=a_{1}+(\mu+(\beta-1) \alpha)(a) .
$$

We thus obtain $A_{1}=(1+\alpha) A$ and $\mu_{1}: A_{1} \rightarrow W$ such that $\left[a_{1}, b\right]=a_{1}+\mu_{1}\left(a_{1}\right)$ with $\mu_{1}\left(a_{1}\right)=\mu(a)+(\beta-1) \alpha(a)$. Regarding $\alpha, \mu, \mu_{1}$ as maps $(A+W) / W \rightarrow W$, we have $\mu_{1}=\mu+(\beta-1) \alpha$. By making a suitable choice of subspace $A_{1}$, we can thus 
replace $\mu$ by $\mu+\gamma$ for any $\gamma: A \rightarrow W$ for which there exists $\alpha$ with $(\beta-1) \alpha=\gamma$, that is, any $\gamma$ such that $\gamma(A) \subseteq(\beta-1) W$. Since $(\beta-1)$ acts invertibly on $W_{0}+W_{*}$, we can choose $A$ such that $\mu(A) \subseteq W_{1}$. As $W_{1}=\left[W_{1}, b\right] \subseteq L^{\prime}$, for this choice of $A$, we have $a=[a, b]-\mu(a) \in L^{\prime}$ for all $a \in A$, so $A \subseteq L^{\prime}$ and $A$ complements $W \cap L^{\prime}$ in $L^{\prime}$. By Lemma 6.1, $\left[W, L^{\prime}\right]=0$. We have

$$
\left[\left[a, a^{\prime}\right], b\right]=\left[[a, b], a^{\prime}\right]+\left[a,\left[a^{\prime}, b\right]\right]=\left[a+\mu(a), a^{\prime}\right]+\left[a, a^{\prime}+\mu\left(a^{\prime}\right)\right]=2\left[a, a^{\prime}\right]
$$

since $[W, A]=0$. Since char $F \neq 2,[A, A] \leq W_{*}$.

We now prove $r \leq 1$. Let $U=b^{s L}$. Since $\beta^{i}\left(W_{1}+W_{*}\right)=W_{1}+W_{*}$ for all $i$, $W_{1}+W_{*} \leq U$. Since $\left.[\cdots[A, b], b] \cdots, b\right]+W_{1}=A+W_{1}$ whatever the number of factors, $A \subseteq U$. But as $\left\langle b, A, \beta^{r} W\right\rangle \triangleleft\left\langle b, A, \beta^{r-1} W\right\rangle \triangleleft \cdots \triangleleft\langle b, A, W\rangle=L$, it follows that $U=\left\langle b, A, W_{1}, W_{*}\right\rangle$. Therefore $[W, b] \subseteq W_{1}+W_{*}$, so $r \leq 1$.

We now clearly have $L^{\prime}=A+W_{1}+W_{*}$ and $L^{\prime \prime}=[A, A]$. Also, $W_{0} \triangleleft L$ and $L=W_{0} \oplus\left(W_{1}+W_{*}+A+\langle b\rangle\right)$. Since $L$ has no abelian direct summand, $W_{0}=0$ and $W=W_{1}+W_{*}$.

Now suppose $a \in A, a \neq 0$. Since $a \notin \omega(L)$, there exists $x \in L$ such that $[a, x] \notin x^{s L}$. If $x \notin W+A$, then $x^{s L}=W+A+\langle x\rangle=L$, so $x \in W+A$ and $x^{s L}=\langle x\rangle$. We have $x=w+a^{\prime}$ for some $w \in W$ and $a^{\prime} \in A$. Now $[a, x]=\left[a, a^{\prime}\right]$, so $\left[a, a^{\prime}\right] \notin x^{s L}$ which implies $\left[a, a^{\prime}\right] \neq 0$.

In the above, we still have some freedom of choice for the subspace $A$ and the corresponding map $\mu: A \rightarrow W_{1}$. For any linear map $\alpha: A \rightarrow W_{1}$, the subspace $A_{1}=(1+\alpha) A$ also satisfies our requirements with $\mu$ replaced by $\mu_{1}=\mu+(\beta-1) \alpha$. Let $\bar{\mu}, \bar{\mu}_{1}$ denote the composites of $\mu, \mu_{1}$ with the natural map $W_{1} \rightarrow W_{1} /(\beta-1) W_{1}$. Then $\bar{\mu}=\bar{\mu}_{1}$. Conversely, for any linear map $\mu_{1}: A \rightarrow W_{1}$ such that $\bar{\mu}_{1}=\bar{\mu}$, there exists a linear map $\alpha: A \rightarrow W_{1}$ such that $\mu_{1}-\mu=(\beta-1) \alpha$. Thus by suitable choice of $A_{1}$, we may replace $\mu$ by any $\mu_{1}$ with $\bar{\mu}_{1}=\bar{\mu}$.

Any Lie algebra $L$ with the structure described has Wielandt length 2 and $L / \omega(L)$ non-abelian. Indeed, given the above data, we can construct a Lie algebra, uniquely determined by the data, which has those properties.

THEOREM 9.2. Let $F$ be a field with char $F \neq 2$. Let $W, A$ be vector spaces over $F$ with $\operatorname{dim} A \geq 2$. Let $\beta: W \rightarrow W$ be a non-singular linear transformation with 2 as an eigenvalue. Let $W_{1}=\left\{w \in W \mid(\beta-1)^{r} w=0\right.$ for some $\left.r\right\}$, let $W_{*}$ be the complementary invariant subspace and let $W_{2}=\{w \in W \mid b(w)=2 w\}$. Let $m: A \times A \rightarrow W_{2}$ be a non-singular alternating bilinear map and let $\tau: A \rightarrow$ $W /(\beta-1) W$ be any linear map. Take $B=\langle b\rangle$ a 1-dimensional vector space. Let $\mu$ be any lifting of $\tau$ to a linear map into $W_{1}$. Put $L=W \oplus A \oplus B$ and define the multiplication [, ] $L \wedge L \rightarrow L$ by

$$
\left[w, w^{\prime}\right]=[w, a]=0, \quad\left[a, a^{\prime}\right]=m\left(a, a^{\prime}\right), \quad[w, b]=\beta(w), \quad[a, b]=a+\mu(a)
$$


for all $w, w^{\prime} \in W$ and $a, a^{\prime} \in A$. Then $L$ is a Lie algebra with no non-trivial abelian direct summand, $L^{\prime}=W+A$ is nilpotent of class $2, \omega(L)=W=\mathscr{Z}\left(L^{\prime}\right), L$ has Wielandt length 2 and $L / \omega(L)$ is non-abelian.

PRoof. By definition, $[x, y]=-[y, x]$ for all $x, y \in L$. The Jacobi identity is easily verified. Thus $L$ is a Lie algebra. Clearly, $L^{\prime}=W+A$. Since $m$ is non-singular, for any $a \in A, a \neq 0$, there exists $a^{\prime} \in A$ with $\left[a, a^{\prime}\right] \neq 0$. Thus $L^{\prime \prime}=[A, A] \neq 0$. But $[A, A] \leq W$ and $[W, W+A]=0$. Thus $L^{\prime}$ is nilpotent of class 2. If $a \in A, a \neq 0$ and $w \in W$, then for some $a^{\prime} \in A$, we have $\left[a+w, a^{\prime}\right] \neq 0$. Hence $W=\mathscr{Z}\left(L^{\prime}\right)$. Thus $\omega(L) \leq W$. If $x=b+w+a$ for some $w \in W$ and $a \in A$, then $[W, x]=[W, b]=W$, so $W \leq x^{s L}$. Since $[a+W, x]=a+W$ for all $a \in A, A \leq x^{s L}$. Thus $x^{s L} \geq L^{\prime}$ so $x^{s L} \triangleleft L$. It follows that $W \leq \mathscr{N}_{L}(U)$ for every $U \triangleleft \Delta$ with $U \notin L^{\prime}$. But $W=\mathscr{Z}\left(L^{\prime}\right)$, so $W \leq \mathscr{N}_{L}(U)$ for $U \triangleleft \Delta L$ with $U \leq L^{\prime}$. Thus $W=\omega(L)$. As $L / W$ has the abelian ideal $A+W / W$ of codimension 1 with $b+W$ acting as the identity, $L / W$ is non-abelian of Wielandt length 1 . Finally, as $\mathscr{Z}(L)=0, L$ has no non-trivial abelian direct summand.

The situation in characteristic 2 is a little different.

THEOREM 9.3. Suppose char $F=2$. Let $L$ be a completely soluble Lie algebra over $F$ with Wielandt length 2 . Suppose $L / \omega(L)$ is non-abelian. Suppose $L$ has no nontrivial abelian direct summand. Then there exist subspaces $W_{0}, W_{\bullet}, A$ and an element $b$ such that, putting $W=W_{0} \oplus W_{\text {., }}$ the following conditions are satisfied.

(1) As vector space, $L=W \oplus A \oplus\langle b\rangle$.

(2) $[W, W+A]=0$.

(3) Setting $\beta(w)=[w, b]$ defines a linear map $\beta: W \rightarrow W$ with $\beta^{2}\left(W_{0}\right)=0$ and $\beta\left(W_{\bullet}\right)=W_{\bullet}$.

(4) Put $W_{1}=\left\{w \in W \mid(\beta-1)^{n} w=0\right.$ for some $\left.n\right\}$. Then for all $a \in A$, $[a, b]=a+\mu(a)$ where $\mu(a) \in W_{1}$.

(5) $[A, A]=L^{\prime \prime}=\{w \in W \mid \beta(w)=0\}$.

(6) For all $a \in A, a \neq 0$, there exists $a^{\prime} \in A$ with $\left[a, a^{\prime}\right] \neq 0$.

Proof. As in the proof of Theorem 9.1, we obtain spaces $W, A$ and an element $b$ giving maps $\beta: W \rightarrow W$ and $\mu: A \rightarrow W$. As before, we get a decomposition $W=W_{0} \oplus W_{1} \oplus W_{*}$. We put $W_{0}=W_{1}+W_{*}$. As before, we can adjust the choice of $A$ so that we have $\mu(A) \subseteq W_{1}$. Also as before, we have $\left[\left[a, a^{\prime}\right], b\right]=2\left[a, a^{\prime}\right]$, but as char $F=2$, we have $[A, A] \subseteq\{w \in W \mid \beta(w)=0\} \subseteq W_{0}$.

We now prove $\beta\left(W_{0}\right) \subseteq[A, A]$. Let $U=b^{s L}$. As before, we see that $A+W_{\bullet} \subseteq U$, and it follows that $U=\left\langle A,[A, A], W_{\bullet}, b\right\rangle$. Hence, if $w \in W_{0}$, then $\beta(w) \in[A, A]$.

If $\beta(w)=0$ and $w \notin[A, A]$, then $\langle w\rangle$ is an abelian direct summand of $L$. Therefore $\{w \in W \mid \beta(w)=0\}=[A, A]$. As before, for any non-zero $a \in A$, there exists 
$a^{\prime} \in A$ with $\left[a, a^{\prime}\right] \neq 0$.

As in the case char $F \neq 2$, given the spaces and maps as above, we can construct a Lie algebra with the required properties. We leave the details to the reader.

We now consider algebras $L$ with both $\omega(L)$ and $L / \omega(L)$ abelian.

THEOREM 9.4. Suppose $L$ has Wielandt length 2 and both $\omega(L)$ and $L / \omega(L)$ are abelian. Suppose $L$ has no non-trivial abelian direct summand. Then there exist subspaces $W_{0}, \ldots, W_{k}, A$ of $L, W_{\bullet}=W_{1} \oplus \cdots \oplus W_{k}$, such that

(1) $\omega(L)=W_{0} \oplus W_{\text {. }}$

(2) $L=\omega(L) \oplus A$ as vector space.

(3) For all $a \in A$ and all $i$, either $\left[W_{i}, a\right]=0$ or $\left[W_{i}, a\right]=W_{i}$.

(4) $\left[W_{0}, A\right]=0$ and $\left[W_{0}, A\right]=W_{\text {. }}$

(5) $W_{0}=[A, A]$

(6) If $a \in A, a \neq 0$, then $[L, a] \neq 0$.

(7) Either $\operatorname{dim} A>1$ or there exists $a \in A$ such that the action $w \mapsto[w, a]$ is not a scalar transformation of $W$.

Proof. Put $W=\omega(L)$. Then $W$ is an $L / W$-module. Since $L / W$ is abelian, $W$ is the direct sum $W=W_{0} \oplus \cdots \oplus W_{k}$ of submodules $W_{i}$, each of which has only one type of composition factor. (See for example, Barnes [2].) Let $W_{0}$ be the component of the trivial composition factors and let $W_{0}$ be the sum of the components with non-trivial composition factors. By Barnes [1, Theorem 1], $H^{2}\left(L / A, W_{\bullet}\right)=0$, so $L / W_{0}$ splits over $W / W_{0}$. Thus there exists a subspace $A$ such that $L=W \oplus A$ as vector space and $[A, A] \subseteq W_{0}$. We have $\left[W_{0}, A\right]=W_{\text {. }}$. Suppose $a \in A$. Let $\bar{a}: W \rightarrow W$ be the linear transformation given by $\bar{a}(w)=[w, a]$ and let $W=W_{0}(a) \oplus W_{0}(a)$ be the decomposition of $W$ into null and non-null components with respect to $\bar{a}$. Suppose $a \neq 0$ and $w \in W$. Put $u=w+a$. Then $\langle W, u\rangle \triangleleft L$ and $u^{s L}=u^{s(W, u\rangle}$, so it follows that $u^{s L}=\left\langle W_{\bullet}(a), u\right\rangle$. If $w \in W_{0}(a)$, then $[w, a]=[w, u] \in u^{s L}$, so $[w, a]=0$. Thus for each $W_{i}$, either $\left[W_{i}, a\right]=0$ or $W_{i}$ has no trivial composition factor as $\langle a\rangle$-module and so $\left[W_{i}, a\right]=W_{i}$. In particular, $\left[W_{0}, A\right]=0$. For each of the other components, there is some $a \in A$ which acts non-trivially, so $\left[W_{\bullet}, A\right]=W_{\bullet}$.

If $w \in W_{0}$ and $w \notin[A, A]$, then $\langle w\rangle$ is an abelian direct summand of $L$. Therefore $W_{0}=[A, A]$. If $a \in A$ and $[L, a]=0$, then $\langle a\rangle$ is an abelian direct summand, so $a=0$. Clearly, $L \neq W$, so $\operatorname{dim} A \geq 1$. If $A=\langle a\rangle$, and $\bar{a}$ is scalar, then $\omega(L)=L$ contrary to assumption.

Clearly, if we are given vector spaces $W_{0}, \ldots, W_{k}, A, W=W_{0} \oplus \cdots \oplus W_{k}$, and, for all $a \in A$, a linear transformation $\bar{a}: W \rightarrow W$, and an alternating bilinear map $m: A \times A \rightarrow W_{0}$ such that

(1) $W \neq 0$ and $A \neq 0$; 
(2) for all $a, a^{\prime} \in A, \bar{a} \bar{a}^{\prime}=\bar{a}^{\prime} \bar{a}$;

(3) for all $a \in A$ and all $i$, either $\bar{a}\left(W_{i}\right)=0$ or $\bar{a}\left(W_{i}\right)=W_{i}$;

(4) for all $a \in A, \bar{a}\left(W_{0}\right)=0$;

(5) for all $i \neq 0$, there exists $a \in A$ such that $\bar{a}\left(W_{i}\right)=W_{i}$;

(6) $m(A, A)=W_{0}$;

(7) for all $a \in A, a \neq 0$, either $\bar{a} \neq 0$ or there exists $a^{\prime} \in A$ such that $m\left(a, a^{\prime}\right) \neq 0$;

(8) if $\operatorname{dim} A=1$ and $a \in A, a \neq 0$, then $\bar{a}$ is not scalar

then we can construct a Lie algebra $L$ as in Theorem 9.4 by setting $L=W \oplus A$, $\left[w, w^{\prime}\right]=0,\left[a, a^{\prime}\right]=m\left(a, a^{\prime}\right)$ and $[w, a]=\bar{a}(w)$ for all $w, w^{\prime} \in W$ and $a, a^{\prime} \in A$.

\section{Lie algebras with Wielandt length $>2$}

We have seen (Theorem 6.2) that in the Wielandt series

$$
0<\omega_{1}(L)<\cdots<\omega_{n-1}(L)<\omega_{n}(L)=L
$$

only the last $L / \omega_{n-1}(L)$ or second last $\omega_{n-1}(L) / \omega_{n-2}(L)$, but not both, of the quotients $\omega_{i}(L) / \omega_{i-1}(L)$ can be non-abelian. By Theorem 6.2, the second last non-abelian can only occur if the field $F$ is not algebraically closed. In proving Theorem 8.7 , we showed that we can have an arbitrarily long Wielandt series with non-abelian final quotient. We now show that we can have arbitrarily long Wielandt series with nonabelian penultimate quotient.

If $S$ is the free Lie algebra generated by $x_{1}, \ldots, x_{k}$, then $S$ is positively graded with $S_{i}$ the space spanned by the products of $i$ factors. Any linear transformation $\alpha_{1}: S_{1} \rightarrow S_{1}$ has a unique extension to a derivation $\alpha: S \rightarrow S$ of degree 0 . We may form the quotient by the ideal $\sum_{i>n} S_{i}$ to form the free nilpotent algebra $N$ of class $n$ which inherits the derivation $\alpha$. We use this to produce our example. Put $N_{r}^{*}=\sum_{i \geq r} N_{r}$

EXAMPLE 10.1. Let $N$ be the free nilpotent Lie algebra of class $n$ on the generators $x_{1}, \ldots, x_{k}$. Let $\alpha, \beta$ be the derivations of $N$ obtained by extending the maps $\alpha_{1}, \beta_{1}$ : $N_{1} \rightarrow N_{1}$ where $\alpha_{1}$ is any map having no eigenvalue in $F$, and $\beta_{1}$ is the identity. Let $L$ be the split extension of $N$ by the abelian algebra $A=\langle\alpha, \beta\rangle$. Then $\omega_{n-1}(L)=$ $\mathscr{Z}_{n-1}(N)=N_{2}^{*}, \omega_{n}(L) / \omega_{n-1}(L)=\left\langle\beta, N_{1}\right\rangle$ is non-abelian, and $\omega_{n+1}(L)=L$.

ProOF. Suppose $U \triangleleft \Delta$ and that $U$ is not an ideal. Then $U<M$ for some maximal ideal $M$. But $M=((\lambda \alpha+\mu \beta), N\rangle$ for some $\lambda, \mu \in F$, not both zero, since $L^{\prime}=N$. But $\lambda \alpha+\mu \beta$ acts invertibly on $N_{\mathrm{l}}$, so $M^{\prime}=N$ and it follows that $U \leq N$. It follows that $\omega_{i}(L)=\mathscr{Z}_{i}(N)=N_{n-i+1}^{*}$ for $i=1, \ldots, n-1$. The result follows by Theorem 6.2. 
Acknowledgements This work began as an MSc project [9] at the Australian National University by the second author, who thanks R. Bryce and J. Cossey for their continued help for the duration of the project. It has been generalised and extended by the first author.

\section{References}

[1] D. W. Barnes, 'On the cohomology of soluble Lie algebras', Math. Z. 101 (1967), 343-349.

[2] _ - 'Sortability of representations of Lie algebras', J. Algebra 27 (1973), 486-490.

[3] R. Brandl, S. Franciosi and F. de Giovanni, 'On the Wielandt length of infinite soluble groups', Glasgow Math. J. 32 (1990), 121-125.

[4] R. Bryce and J. Cossey, 'The Wielandt subgroup of a finite group', J. London Math. Soc. 40 (1989), 244-256.

[5] R. Bryce, J. Cossey and E. Ormerod, 'A note on p-groups with power automorphisms', Glasgow Math. J. 34 (1992), 327-332.

[6] A. Camina, 'The Wielandt length of finite groups', J. Algebra 15 (1970), 142-148.

[7] C. Casolo, 'Soluble groups with finite Wielandt length', Glasgow Math. J. 31 (1989), 329-334.

[8] — 'Wielandt series and defects of subnormal subgroups in finite soluble groups', Rend. Sem. Mat. Univ. Padova 87 (1992), 93-104.

[9] D. Groves, The Wielandt ideal of a Lie algebra (M.Sc. Thesis, Australian National University, 1998).

[10] B. Hartley, 'Locally nilpotent ideals of a Lie algebra', Proc. Cambridge Philos. Soc. 63 (1967), 257-272.

[11] N. Jacobson, Lie algebras (Interscience, New York, 1962).

[12] E. Schenkman, 'A theory of subinvariant Lie algebras', Amer. J. Math. 73 (1951), 453-474.

[13] I. Stewart, Subideals of Lie algebras (Ph.D. Thesis, University of Warwick, 1969).

[14] H. Strade and R. Farnsteiner, Modular Lie algebras and their representations, Monographs Textbooks Pure Appl. Math. 116 (Marcel Dekker, New York, 1988).

[15] W. Tuck, Frattini theory for Lie algebras (Ph.D. Thesis, University of Sydney, 1969).

[16] H. Wielandt, 'Über den Normalisator der subnormalen Untergruppen', Math. Z. 69 (1958), 463465.

[17] H. Zassenhaus, 'On trace bilinear forms on Lie-algebras', Proc. Glasgow Math. Assoc. 4 (1959), 62-72.

1 Little Wonga Rd

Cremorne NSW 2090

Australia

e-mail: donb@netspace.net.au
Department of Mathematics

School of Advanced Studies

Australian National University

ACT 0200

Australia

Current address:

Mathematical Institute

24-29 St. Giles

Oxford, OX1 3LB

UK

e-mail: grovesd@maths.ox.ac.uk 